

Chemical Communications rsc.li/chemcomm

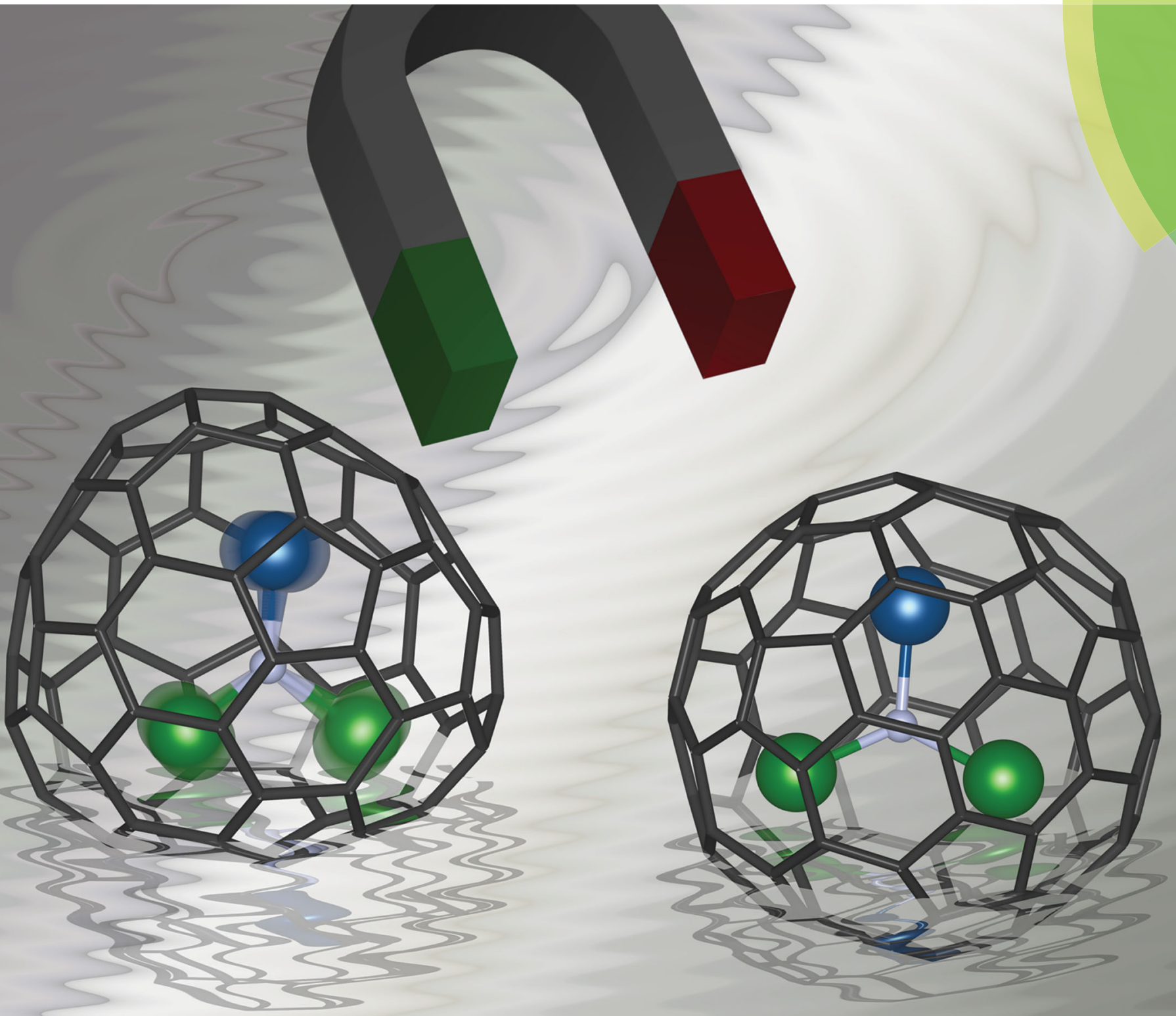

ISSN 1359-7345

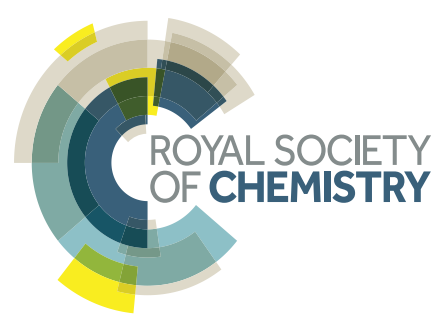


Check for updates

Cite this: Chem. Commun., 2018, 54,9730

Received 24th June 2018, Accepted 24th July 2018

DOI: $10.1039 / \mathrm{c} 8 \mathrm{cc} 05029 \mathrm{e}$ rsc.li/chemcomm

\section{Strong carbon cage influence on the single molecule magnetism in Dy-Sc nitride clusterfullerenes $\dagger$}

\author{
Christin Schlesier, ${ }^{a}$ Lukas Spree, ${ }^{a}$ Aram Kostanyan, ${ }^{\text {b }}$ Rasmus Westerström, ${ }^{\text {b }}$ \\ Ariane Brandenburg, (D) ${ }^{a}$ Anja U. B. Wolter, ${ }^{a}$ Shangfeng Yang, (DD ${ }^{c}$ \\ Thomas Greber (D) ${ }^{b}$ and Alexey A. Popov (D) *a
}

\begin{abstract}
Magnetic properties of endohedral metallofullerenes with nitride clusters $\mathrm{DySC}_{2} \mathrm{~N}$ and $\mathrm{Dy}_{2} \mathrm{ScN}$ and different carbon cages are studied by SQUID magnetometry. All molecules behave as single molecule magnets (SMMs) and exhibit magnetic hysteresis. It is found that the blocking temperature of magnetization and relaxation times strongly depend on the fullerene cage, with the $C_{80}-I_{h}$ isomer offering the best SMM properties.
\end{abstract}

The ability of fullerenes to stabilize unconventional species inside the protective shell of their carbon cages resulted in a plethora of different classes of endohedral metallofullerenes (EMFs). ${ }^{1}$ The endohedral metal ions can preserve some of their physical properties (such as spin states) inside the carbon cage, whereas intramolecular interactions with other ions in the cluster as well as the electron transfer to the carbon cage can lead to the development of new properties. In 2012 it was found that $\mathrm{DySc}_{2} \mathrm{~N} @ \mathrm{C}_{80}-I_{\mathrm{h}}$ exhibits slow relaxation of magnetization in a finite magnetic field of $0.2 \mathrm{~T}^{2}$ In zero field, the magnetization of $\mathrm{DySc}_{2} \mathrm{~N} @ \mathrm{C}_{80}-I_{\mathrm{h}}$ relaxes fast via quantum tunnelling of magnetization (QTM), resulting in a characteristic butterfly shape of the magnetic hysteresis typical for many single-ion magnets (SIMs). ${ }^{3}$ Further studies revealed single molecule magnetism (SMM) in other lanthanide-based EMFs, including clusterfullerenes with nitride, ${ }^{4}$ sulfide, ${ }^{5}$ carbide,${ }^{5,6}$ or cyanide units, ${ }^{7}$ as well as in dimetallofullerenes. ${ }^{8}$ The SMM properties were found to depend substantially on the choice of the metal (Dy gives the longest relaxation times) and the endohedral cluster composition. For instance, QTM at zero field is suppressed in

\footnotetext{
${ }^{a}$ Leibniz Institute for Solid State and Materials Research (IFW), D-01069 Dresden, Germany.E-mail: a.popov@ifw-dresden.de

${ }^{b}$ Physik-Institut der Universität Zürich, Winterthurerstr. 190, CH-8057 Zürich, Switzerland

${ }^{c}$ Hefei National Laboratory for Physical Sciences at Microscale, CAS Key Laboratory of Materials for Energy Conversion, Department of Materials Science and Engineering, University of Science and Technology of China, Hefei 230026, China $\dagger$ Electronic supplementary information (ESI) available: Mass-spectra and determination of relaxation times. See DOI: 10.1039/c8cc05029e
}

$\mathrm{Dy}_{2} \mathrm{ScN} @ \mathrm{C}_{80}-\mathrm{I}_{\mathrm{h}}$ due to the ferromagnetic exchange and dipolar interactions of the two Dy ions. ${ }^{4 a}$

Among the various lanthanide clusterfullerenes, $\mathrm{DySc}_{2} \mathrm{~N}$ and $\mathrm{Dy}_{2} \mathrm{ScN}$ clusterfullerenes showed the best SMM performance presumably due to the strong ligand field created by the nitride ion located at the short distance from the lanthanide. ${ }^{4 b, 5,9}$ Studies of the magnetic properties of nitride clusterfullerenes have been limited so far to the $\mathrm{C}_{80}-I_{\mathrm{h}}$ cage because the corresponding EMFs are usually produced in much higher yields and constitute $c a .80 \%$ of the nitride clusterfullerenes mixtures. Yet, the arc-discharge synthesis of nitride clusterfullerenes affords a variety of other isolable carbon cages, from $\mathrm{C}_{68}$ to $\mathrm{C}_{88}$, as well as another cage isomer of $\mathrm{C}_{80}$, with $D_{5 \mathrm{~h}}$ symmetry (the Gd-Sc and Dy-Sc systems have been studied in most detail). ${ }^{10}$ Due to the much lower production yield their properties remain largely unexplored. It is not clearly understood if the fullerene cage is merely a container for magnetic species, or if it plays an active role in the relaxation of magnetization. For $\mathrm{Dy}_{2} \mathrm{~S}$ - and TbCN-clusterfullerenes the variation of the carbon cage was found to affect the magnetic properties. ${ }^{5,7 b}$ In this Communication, we show that the magnetic properties of several Dy-Sc nitride clusterfullerenes strongly depend on their fullerene cages and that the most symmetric $\mathrm{C}_{80}-I_{\mathrm{h}}$ fullerene provides the best SMM properties of the endohedral clusters.

The structures chosen for this study (Fig. 1), DySc 2 N@C $\mathrm{C}_{68^{-}}$ $D_{3}(6140), \mathrm{Dy}_{2} \mathrm{ScN} @ \mathrm{C}_{84}-C_{\mathrm{s}}(51365), \mathrm{DySc}_{2} \mathrm{~N} @ \mathrm{C}_{80}-D_{5 \mathrm{~h}}(6)$, and $\mathrm{Dy}_{2} \mathrm{ScN} @ \mathrm{C}_{80}-D_{5 \mathrm{~h}}(6), \ddagger$ have been obtained using arc-discharge synthesis followed by HPLC separation as described in detail earlier. ${ }^{10 b, d}$ This set of EMFs allows to compare the influence of the carbon cage isomerism $\left(I_{\mathrm{h}}\right.$ versus $\left.D_{5 \mathrm{~h}}\right)$ as well as the size of the fullerene cage $\left(\mathrm{C}_{68}\right.$ versus $\mathrm{C}_{80}$ versus $\left.\mathrm{C}_{84}\right)$.

In $\mathrm{DySc}_{2} \mathrm{~N} @ \mathrm{C}_{68}$, the $D_{3}$-symmetric fullerene cage violates the isolated pentagon rule, as it features three adjacent pentagon pairs coordinated by metal atoms of the endohedral nitride cluster. The cluster is therefore tightly fixed inside the carbon cage. $\mathrm{Dy}_{2} \mathrm{ScN} @ \mathrm{C}_{84}$ with the $C_{\mathrm{s}}(51365)$ fullerene cage has one pentagon adjacency and thus also violates the IPR. Computational studies 
a

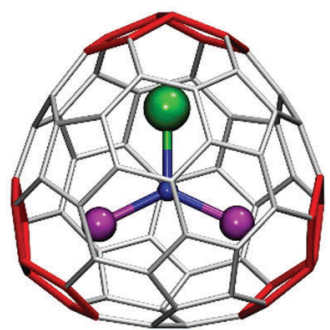

C



b



d

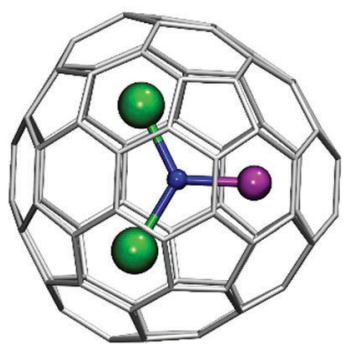

Fig. 1 Molecular structures of Dy-Sc nitride clusterfullerenes studies in this work: (a) DySc${ }_{2} N @ C_{68}-D_{3}(6140)$, the $C_{3}$ axis of the cage is perpendicular to the paper plane; (b) $\mathrm{Dy}_{2} \mathrm{ScNaC}_{84}-\mathrm{C}_{\mathrm{s}}(51365)$, the symmetry plane of the cage

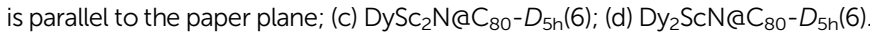
Dy atoms are green, Sc atoms are magenta, $\mathrm{N}$ is blue, carbon cages are grey except for adjacent pentagon pairs shown in red. In (c) and (d), the plane of the cluster is parallel to one of the $\sigma_{\mathrm{h}}$ planes of the cage, whereas the $C_{5}$ axis is oriented vertically and is in the plane of the paper. All structures are based on DFT calculations of $\mathrm{Y}-\mathrm{Sc}$ analogues.

showed that the structure in which the pentagon pair is coordinated by the bigger metal is more stable. However, the energy of the conformer with a Sc-coordinated pentagon pair is only slightly higher, and thus both structures are likely to be present. The pentagon adjacency fixes the position of the metal cluster, which therefore cannot rotate freely in $\mathrm{DySc}_{2} \mathrm{~N} @ \mathrm{C}_{68}$ and $\mathrm{Dy}_{2} \mathrm{ScN} @ \mathrm{C}_{84}$.

The $\mathrm{C}_{80}-D_{5 \mathrm{~h}}$ cage resembles the $\mathrm{C}_{80}-\mathrm{I}_{\mathrm{h}}$ counterpart. Both isomers can be described as built from identical hemispheres, but rotated with respect to each other at different angles. The NMR studies of Lu-Sc nitride clusterfullerenes showed that the nitride cluster inside the $\mathrm{C}_{80}-D_{5 \mathrm{~h}}$ cage rotates freely at room temperature, similar to the rotation inside the $\mathrm{C}_{80}-I_{\mathrm{h}}$ cage. ${ }^{11}$ Thus, disordered positions of the endohedral cluster are to be expected in $\mathrm{DySc}_{2} \mathrm{~N} @ \mathrm{C}_{80}-D_{5 \mathrm{~h}}$ and $\mathrm{Dy}_{2} \mathrm{ScN} @ \mathrm{C}_{80}-D_{5 \mathrm{~h}}$, and Fig. 1 shows one of the lowest energy configurations determined by DFT calculations. ${ }^{10 b}$

As a measure of the SMM performance we use several parameters (Table 1), which can be obtained from DC SQUID magnetometry (the low yield of these minor structures precludes the isolation of amounts sufficient for AC magnetometry). First of all, magnetic hysteresis is a clear indication that the sample behaves as an SMM (Fig. 2). The width of the hysteresis and the temperature at which it is closing are characteristic parameters of a given SMM. Another important characteristic parameter of the SMM is the blocking temperature of magnetization, $T_{\mathrm{B}}$. Here we adopt the definition of $T_{\mathrm{B}}$ based on the divergence of the magnetic susceptibility of the SMM measured during cooling the sample in a field (FC) and for the sample cooled in zero field (ZFC). $T_{\mathrm{B}}$ is then defined as the temperature of the
Table 1 Selected SMM parameters in Dy-Sc nitride clusterfullerenes

\begin{tabular}{llcl}
\hline EMF & $T_{\mathrm{B}}{ }^{a}, \mathrm{~K}$ & $T_{\mathrm{B} 100}{ }^{b}, \mathrm{~K}$ & $H_{\mathrm{c}}, T(\text { at } 2 \mathrm{~K})^{c}$ \\
\hline DySc $_{2} \mathrm{~N} @ \mathrm{C}_{68}-D_{3}(6140)$ & 3.8 & 2.3 & \\
DySc $_{2} \mathrm{~N} @ \mathrm{C}_{80}-D_{5 \mathrm{~h}}(6)$ & 5.9 & 3.6 & \\
DySc $_{2} \mathrm{~N} @ \mathrm{C}_{80}-I_{\mathrm{h}}(7)$ & 7.0 & 4.6 & \\
& & & \\
Dy $_{2} \mathrm{ScN} @ \mathrm{C}_{80}-D_{5 \mathrm{~h}}(6)$ & 5.3 & 2.6 & 0.48 \\
$\mathrm{Dy}_{2} \mathrm{ScN} @ \mathrm{C}_{80}-I_{\mathrm{h}}(7)$ & 8.0 & 5.0 & 0.70 \\
$\mathrm{Dy}_{2} \mathrm{ScN} @ \mathrm{C}_{84}-C_{\mathrm{s}}(51365)$ & 3.3 & $\approx 1.8$ & 0.11
\end{tabular}

${ }^{a} T_{\mathrm{B}}$ measured in the field of $0.2 \mathrm{~T}$ with a sweep rate of $5 \mathrm{~K} \mathrm{~min}^{-1}$. ${ }^{b} T_{\mathrm{B} 100}$ is estimated from the temperature dependence of relaxation times (in zero field for $\mathrm{Dy}_{2} \mathrm{ScN}$-EMFs, and in the field of $0.2 \mathrm{~T}$ for DySc $_{2} \mathrm{~N}$-EMFs). ${ }^{c}$ Coercive field $H_{\mathrm{c}}$ is measured at $2 \mathrm{~K}$ with an average sweep rate of $2.9 \mathrm{mT} \mathrm{s}^{-1}$.

maximum of the peak observed in $\chi_{\mathrm{ZFC}}$ (measured in a field of $0.2 \mathrm{~T}$ with a sweep rate of $5 \mathrm{~K} \mathrm{~min}^{-1}$ in this work; note that the use of a different DC field or a temperature sweep rate may give somewhat different $T_{\mathrm{B}}$ values $^{3 b}$ ). In these measurement conditions, the magnetic relaxation time at $T_{\mathrm{B}}$ is $c a .10$ seconds. At higher temperature, the relaxation becomes fast on the time scale of the experiment, and the two curves coincide. In this definition, $T_{\mathrm{B}}$ is close to the temperature at which magnetic hysteresis is closing, but the latter also depends on the sweep rate. The temperature at which the relaxation time is equal $100 \mathrm{~s}$ is another common parameter denoted here as $T_{\mathrm{B} 100}$. Finally, magnetic relaxation times and their evolution with temperature provide information on the SMM performance. Here relaxation times are measured by first magnetizing the sample to saturation, followed by sweeping the field as fast as possible to the desired value (zero field or $0.2 \mathrm{~T}$ ). Then the decay of the magnetization is recorded, and the resulting decay curves are fitted with a stretched exponential. Due to the finite sweep rates and the time necessary for the stabilization of the field, this procedure gives reliable values for relaxation times longer than $100 \mathrm{~s}$, whereas shorter relaxation times may be somewhat overestimated. A detailed discussion of the relaxation time determination is given in ref. $4 d$.

Fig. 2 shows the magnetization curves of the samples measured at low temperature. All samples exhibit magnetic hysteresis, showing that SMM properties are intrinsic to Dy-Sc nitride clusterfullerenes. However, the width of the magnetic hysteresis and the temperature at which it is closing varies strongly within the series. Both $\mathrm{DySc}_{2} \mathrm{~N} @ \mathrm{C}_{68}$ and $\mathrm{DySc}_{2} \mathrm{~N} @ \mathrm{C}_{80}-D_{5 \mathrm{~h}}$ show a butterfly hysteresis shape with a fast relaxation near zero magnetic field, but the width of the hysteresis is much narrower for the DySc $_{2} \mathrm{~N} @ \mathrm{C}_{68}$. Likewise, the $T_{\mathrm{B}}$ value of $\mathrm{DySc}_{2} \mathrm{~N} @ \mathrm{C}_{68}$ is only $3.8 \mathrm{~K}$, which is much lower than the $T_{\mathrm{B}}$ value of $5.9 \mathrm{~K}$ in DySc $_{2} \mathrm{~N} @ \mathrm{C}_{80}-D_{5 \mathrm{~h}}$. For comparison, the blocking temperature of the magnetization of $\mathrm{DySc}_{2} \mathrm{~N} @ \mathrm{C}_{80}-I_{\mathrm{h}}$ measured in the same conditions is $7 \mathrm{~K}$. Among the studied $\mathrm{DySc}_{2} \mathrm{~N}$-clusterfullerenes, DySc $_{2} \mathrm{~N} @ \mathrm{C}_{80}-I_{\mathrm{h}}$ also shows the broadest hysteresis at $2 \mathrm{~K}$ (see Fig. 2c for a comparison).

A similar behavior is found in the series of $\mathrm{Dy}_{2} \mathrm{ScN}$ clusterfullerenes. Dy $\mathrm{D}_{2} \mathrm{ScN} @ \mathrm{C}_{84}$ has the lowest blocking temperature, $T_{\mathrm{B}}=3.3 \mathrm{~K}$, and the narrowest magnetic hysteresis. In $\mathrm{Dy}_{2} \mathrm{ScN} @ \mathrm{C}_{80}-D_{5 \mathrm{~h}}$, the blocking temperature is increased to 

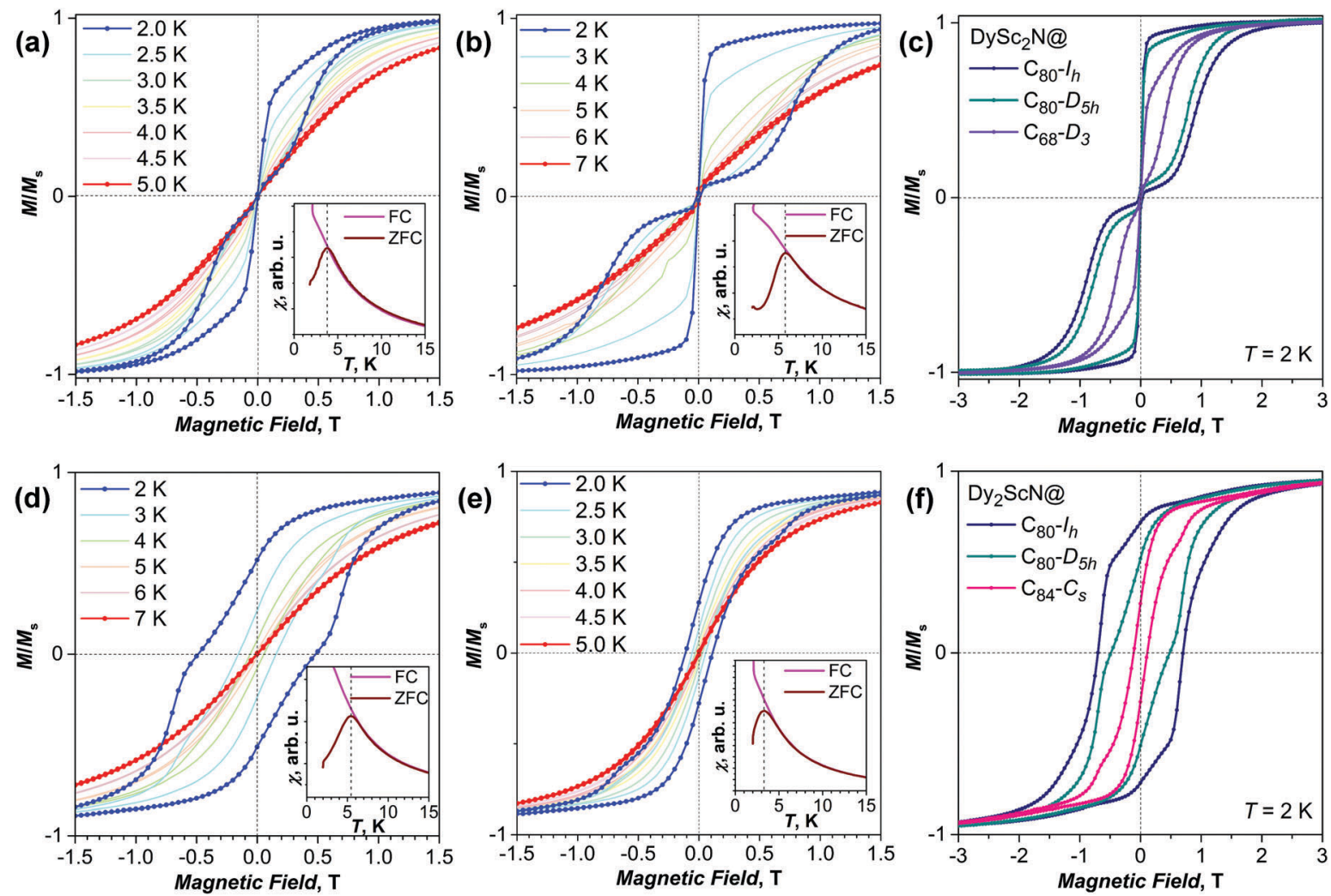

Fig. 2 Low temperature magnetization of Dy-Sc nitride clusterfullerenes: (a) DySc${ }_{2} \mathrm{NaC}_{68}-D_{3}$; (b) DySc${ }_{2} N a C_{80}-D_{5 h}$; (c) comparison of magnetic

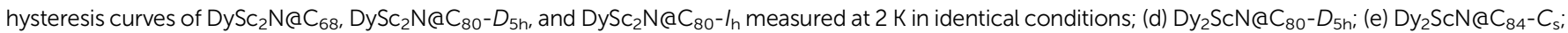
(f) comparison of magnetic hysteresis curves of $\mathrm{Dy}_{2} \mathrm{ScNaC}{ }_{84}, \mathrm{Dy}_{2} \mathrm{ScN} \mathrm{SC}_{80}-D_{5 h}$, and $\mathrm{Dy}_{2} \mathrm{ScNaC}_{80}-I_{\mathrm{h}}$ measured at $2 \mathrm{~K}$ in identical conditions. The insets in (a), (b), (d), and (e) show determination of the blocking temperature of magnetization $T_{\mathrm{B}}$ from temperature dependence of magnetic susceptibility (FC - field cooled, ZFC - zero-field cooled). Magnetic field sweep rate is $2.9 \mathrm{mT} \mathrm{s}^{-1}$, temperature sweep rate is $5 \mathrm{~K} \mathrm{~min}^{-1}$.

$5.3 \mathrm{~K}$, and the magnetic hysteresis is considerably broader. But the SMM parameters of the $D_{5 \mathrm{~h}}$ isomer are inferior to those of $\mathrm{Dy}_{2} \mathrm{ScN} @ \mathrm{C}_{80}-I_{\mathrm{h}}$, which exhibits the $T_{\mathrm{B}}$ value of $8 \mathrm{~K}^{4 b}$

Thus, both for the $\operatorname{DySc}_{2} \mathrm{~N}$ and $\mathrm{Dy}_{2} \mathrm{ScN}$ cluster series, we conclude that the $\mathrm{C}_{80}-I_{\mathrm{h}}$ cage gives the best SMM properties, the $\mathrm{C}_{80}-D_{5 \mathrm{~h}}$ isomer has somewhat lower $T_{\mathrm{B}}$ values and narrower hysteresis, and the non-IPR $\mathrm{C}_{68}-D_{3}$ and $\mathrm{C}_{84}-C_{\mathrm{s}}$ cages give the softest SMMs with $T_{\mathrm{B}}$ values below $4 \mathrm{~K}$ and a narrow hysteresis.

Relaxation times of magnetization $(\tau)$ and their temperature dependence provide another tool to follow the SMM behavior at different temperatures (Fig. 3). For $\mathrm{DySc}_{2} \mathrm{~N} @ \mathrm{C}_{80}-I_{\mathrm{h}}$ our earlier measurements ${ }^{4 d}$ revealed a linear dependence of the $\tau$ values in Arrhenius coordinates between 1.8 and $5 \mathrm{~K}$ (Fig. 3a). Such a dependence corresponds to the Orbach relaxation mechanism:

$$
\tau^{-1}=\tau_{0}{ }^{-1} \exp \left(-U^{\mathrm{eff}} / T\right)
$$

where $U^{\text {eff }}$ is the effective barrier and $\tau_{0}$ is the attempt time, which for $\mathrm{DySc}_{2} \mathrm{~N} @ \mathrm{C}_{80}-I_{\mathrm{h}}$ equals $23.6 \pm 1 \mathrm{~K}$ and $0.6 \pm 0.2 \mathrm{~s}$, respectively. ${ }^{4 d}$ For $\mathrm{DySc}_{2} \mathrm{~N} @ \mathrm{C}_{80}-D_{5 \mathrm{~h}}$, a clear deviation from the linear dependence is found in the same temperature range (Fig. 3a). The data can be equally well described either by a combination of an Orbach relaxation $\left(U^{\text {eff }}=17.7 \pm 0.4 \mathrm{~K}\right.$ and an
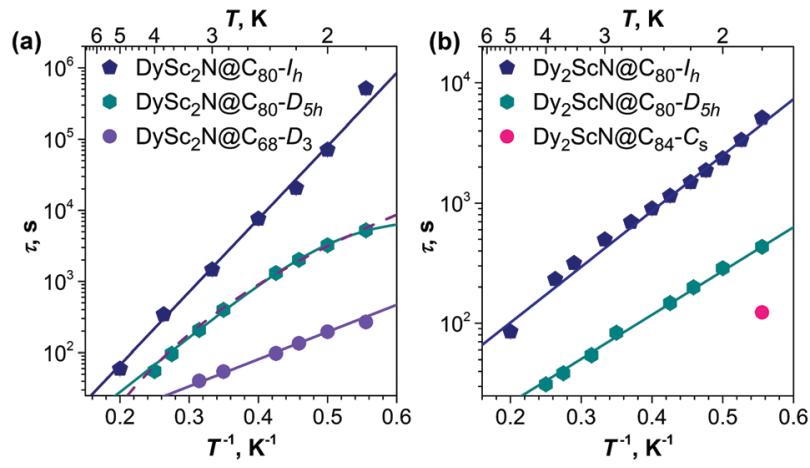

Fig. 3 Temperature dependence of the relaxation times of magnetization measured by DC magnetometry for (a) DySC $\mathrm{C}_{2} \mathrm{~N}$-EMFs in the field of $0.2 \mathrm{~T}$ to avoid QTM, and (b) for Dy ${ }_{2} S c N$-EMFs in zero field. The lines are fits of the experimental data with the model discussed in the text.

attempt time of $\left.\tau_{0}=0.8 \pm 0.1 \mathrm{~s}\right)$ with a temperature-independent quantum tunneling $\left(\mathrm{QTM}, \tau_{\mathrm{QTM}}=0.8 \pm 0.1 \mathrm{~s}\right)$ denoted by a solid line in Fig. 3a, or by a power function of temperature, $\tau^{-1}=A T^{n}$, with $n=5.6 \pm 0.2$ and $A=6.7 \pm 0.8 \times 10^{-6} \mathrm{~s}^{-1} \mathrm{~K}^{-n}$. The power function with such parameters corresponds to the Raman relaxation mechanism with a contribution of optical phonons. ${ }^{12}$ Relaxation times of $\mathrm{DySc}_{2} \mathrm{~N} @ \mathrm{C}_{68}$ between 1.8 and $3 \mathrm{~K}$ can be described 
by the Orbach mechanism with parameters $U^{\text {eff }}=7.6 \pm 0.4 \mathrm{~K}$ and $\tau_{0}=4.1 \pm 0.9 \mathrm{~s}$. Thus, for all three DySc ${ }_{2} \mathrm{~N}$-EMFs the Orbach relaxation with a small barrier appears to be the main relaxation mechanism at low temperatures. Since the barrier is much smaller than expected crystal-field splitting of the Dy ${ }^{6} \mathrm{H}_{15 / 2}$ manifold, ${ }^{9 a}$ the observed barriers have to be assigned to lowfrequency molecular vibrations such as frustrated rotations of the endohedral cluster. ${ }^{4 d, 5,8}$

The low-temperature relaxation of dinuclear Dy-EMFs follows the Orbach mechanism via the exchange/dipolar excited state. That is, exchange and dipolar interactions favour the ferromagnetic (FM) coupling of two Dy spins in the magnetic ground state, whereas the first excited state is ascribed to the antiferromagnetic (AFM) coupling of the moments. ${ }^{4 a, 5}$ For $\mathrm{Dy}_{2} \mathrm{ScN} @ \mathrm{C}_{80}-I_{\mathrm{h}}$, the energy difference between the FM and AFM states has been estimated as $U^{\text {eff }}=10.7 \pm 0.3 \mathrm{~K}$ with an attempt time of $\tau_{0}=11.9 \pm 1.5 \mathrm{~s}^{4 b}$ For $\mathrm{Dy}_{2} \mathrm{ScN} @ \mathrm{C}_{80}-D_{5 \mathrm{~h}}$, this study revealed a somewhat lower barrier of $8.4 \pm 0.2 \mathrm{~K}$ and an attempt time of $4.1 \pm 0.3 \mathrm{~s}$. Thus, while the structure of the Dy-Sc nitride cluster is hardly affected, the $D_{5 \mathrm{~h}}$ isomeric cage reduces both the barrier and the attempt time of the Orbach relaxation via the exchange/dipolar excited state. Even shorter relaxation times of $\mathrm{Dy}_{2} \mathrm{ScN} @ \mathrm{C}_{84}$ preclude the detailed exploration of the temperature dependence. At $1.8 \mathrm{~K}$, the relaxation time of this EMF is $123 \mathrm{~s}$, which is considerably shorter than for both $\mathrm{C}_{80}$ cages.

To conclude, the studies of the low-temperature magnetic properties of a series of Dy-Sc nitride clusterfullerenes showed that the SMM properties of these molecules strongly depend on the fullerene cage. The EMFs with the $\mathrm{C}_{80}-I_{\mathrm{h}}$ cage show the best SMM properties, the non-IPR $\mathrm{C}_{68}-D_{3}$ and $\mathrm{C}_{84}-C_{\mathrm{s}}$ fullerenes give the lowest blocking temperature and narrow magnetic hysteresis curves, whereas the EMFs with $\mathrm{C}_{80}-D_{5 \mathrm{~h}}$ cages exhibit an intermediate behaviour. The size of the fullerene cage does not seem to be as important as its shape. The magnetic relaxation rate can be correlated with the freedom of motion of the endohedral cluster inside the fullerene cage. In the molecules, which exhibit free rotation of the cluster at room temperature, the spin-phonon coupling is presumably weaker than in the molecules with the fixed position of the cluster, which leads to the faster relaxation of magnetization in the latter.

The authors acknowledge funding by the European Research Council under the European Union's Horizon 2020 research and innovation programme (grant No 648295 "GraM3”), the Swiss National Science Foundation (proposals 206021_150784 and 200021L_147201), the Swedish Research Council (Grant No. 2015-00455), and Sklodowska Curie Actions co-founding project INCA 600398. We thank Denis Krylov for the help in determination of relaxation times.

\section{Conflicts of interest}

There are no conflicts to declare.

\section{Notes and references}

$\ddagger$ Fullerene cages are denoted following the spiral algorithm. ${ }^{13}$ For the cages with adjacent pentagon pairs (non-IPR), the full numbering system is used. For IPR cages, more common abridged system counting only IPR isomer is employed.

1 (a) A. A. Popov, S. Yang and L. Dunsch, Chem. Rev., 2013, 113, 5989; (b) S. Yang, T. Wei and F. Jin, Chem. Soc. Rev., 2017, 46, 5005; (c) X. Lu, L. Feng, T. Akasaka and S. Nagase, Chem. Soc. Rev., 2012, 41, 7723; (d) A. Rodriguez-Fortea, A. L. Balch and J. M. Poblet, Chem. Soc. Rev., 2011, 40, 3551.

2 R. Westerström, J. Dreiser, C. Piamonteze, M. Muntwiler, S. Weyeneth, H. Brune, S. Rusponi, F. Nolting, A. Popov, S. Yang, L. Dunsch and T. Greber, J. Am. Chem. Soc., 2012, 134, 9840.

3 (a) D. N. Woodruff, R. E. P. Winpenny and R. A. Layfield, Chem. Rev., 2013, 113, 5110; (b) J.-L. Liu, Y.-C. Chen and M.-L. Tong, Chem. Soc. Rev., 2018, 47, 2431; (c) J. Dreiser, J. Phys.: Condens. Matter, 2015, 27, 183203. 4 (a) R. Westerström, J. Dreiser, C. Piamonteze, M. Muntwiler, S. Weyeneth, K. Krämer, S.-X. Liu, S. Decurtins, A. Popov, S. Yang, L. Dunsch and T. Greber, Phys. Rev. B: Condens. Matter Mater. Phys., 2014, 89, 060406; (b) D. S. Krylov, F. Liu, S. M. Avdoshenko, L. Spree, B. Weise, A. Waske, A. U. B. Wolter, B. Büchner and A. A. Popov, Chem. Commun., 2017, 53, 7901; (c) J. Dreiser, R. Westerström, Y. Zhang, A. A. Popov, L. Dunsch, K. Krämer, S.-X. Liu, S. Decurtins and T. Greber, Chem. - Eur. J., 2014, 20, 13536; (d) D. Krylov, F. Liu, A. Brandenburg, L. Spree, V. Bon, S. Kaskel, A. Wolter, B. Buchner, S. Avdoshenko and A. A. Popov, Phys. Chem. Chem. Phys., 2018, 20, 11656.

5 C.-H. Chen, D. S. Krylov, S. M. Avdoshenko, F. Liu, L. Spree, R. Yadav, A. Alvertis, L. Hozoi, K. Nenkov, A. Kostanyan, T. Greber, A. U. B. Wolter and A. A. Popov, Chem. Sci., 2017, 8, 6451.

6 K. Junghans, C. Schlesier, A. Kostanyan, N. A. Samoylova, Q. Deng, M. Rosenkranz, S. Schiemenz, R. Westerström, T. Greber, B. Büchner and A. A. Popov, Angew. Chem., Int. Ed., 2015, 54, 13411.

7 (a) F. Liu, S. Wang, C.-L. Gao, Q. Deng, X. Zhu, A. Kostanyan, R. Westerström, F. Jin, S.-Y. Xie, A. A. Popov, T. Greber and S. Yang, Angew. Chem., Int. Ed., 2017, 56, 1830; (b) F. Liu, C.-L. Gao, Q. Deng, X. Zhu, A. Kostanyan, R. Westerström, S. Wang, Y.-Z. Tan, J. Tao, S.-Y. Xie, A. A. Popov, T. Greber and S. Yang, J. Am. Chem. Soc., 2016, 138, 14764.

8 F. Liu, D. S. Krylov, L. Spree, S. M. Avdoshenko, N. A. Samoylova, M. Rosenkranz, A. Kostanyan, T. Greber, A. U. B. Wolter, B. Büchner and A. A. Popov, Nat. Commun., 2017, 8, 16098.

9 (a) V. Vieru, L. Ungur and L. F. Chibotaru, J. Phys. Chem. Lett., 2013, 4, 3565; (b) Y. Zhang, D. Krylov, M. Rosenkranz, S. Schiemenz and A. A. Popov, Chem. Sci., 2015, 6, 2328; (c) F. Cimpoesu, N. Dragoe, H. Ramanantoanina, W. Urland and C. Daul, Phys. Chem. Chem. Phys., 2014, 16, 11337.

10 (a) S. F. Yang and L. Dunsch, J. Phys. Chem. B, 2005, 109, 12320; (b) T. Wei, F. Liu, S. Wang, X. Zhu, A. A. Popov and S. Yang, Chem. - Eur. J., 2015, 21, 5750; (c) A. L. Svitova, A. A. Popov and L. Dunsch, Inorg. Chem. 2013, 52, 3368; (d) S. F. Yang, A. A. Popov and L. Dunsch, Chem. Commun., 2008, 2885; (e) S. Yang, A. A. Popov and L. Dunsch, J. Phys. Chem. B, 2007, 111, 13659; $(f)$ M. M. Olmstead, H. M. Lee, J. C. Duchamp, S. Stevenson, D. Marciu, H. C. Dorn and A. L. Balch, Angew. Chem., Int. Ed., 2003, 42, 900; $(g)$ B. Q. Mercado, C. M. Beavers, M. M. Olmstead, M. N. Chaur, K. Walker, B. C. Holloway, L. Echegoyen and A. L. Balch, J. Am. Chem. Soc., 2008, 130, 7854.

11 S. Yang, A. A. Popov, C. Chen and L. Dunsch, J. Phys. Chem. C, 2009, 113, 7616.

12 K. N. Shrivastava, Phys. Status Solidi B, 1983, 117, 437.

13 P. Fowler and D. E. Manolopoulos, An Atlas of Fullerenes, Clarendon Press, Oxford, UK, 1995. 